\title{
Waveguide for Bose-Einstein condensates
}

\author{
K. Bongs, S. Burger, S. Dettmer, D. Hellweg, J. Arlt, W. Ertmer, and K. Sengstock \\ Institut für Quantenoptik, Universität Hannover, Welfengarten 1, 30167 Hannover, Germany
}

(Received 25 July 2000; published 12 February 2001)

\begin{abstract}
We report on the creation of Bose-Einstein condensates of ${ }^{87} \mathrm{Rb}$ in a specially designed hybrid, optical dipole and magnetic trap. This trap naturally allows the coherent transfer of matter waves into a pure optical dipole potential waveguide based on a doughnut beam. Specifically, we present studies of the coherence of the ensemble in the hybrid trap and during the evolution in the waveguide by means of an autocorrelation interferometer scheme. We observe a mean-field dominated acceleration in the waveguide on a much longer time scale than in the free three-dimensional expansion.
\end{abstract}

DOI: 10.1103/PhysRevA.63.031602

PACS number(s): 03.75.Fi, 03.75.Dg, 32.80.Pj, 42.50.Vk

The recent realization of Bose-Einstein condensation (BEC) in dilute atomic gases [1] has stimulated extensive studies on degenerate quantum gases. While most of the experimental work so far has concentrated on threedimensional (3D) systems, there is growing interest in systems with lower dimensionality leading to fundamentally different phenomena. The specific properties of onedimensional (1D) quantum gases were recently studied theoretically and have triggered a debate about, e.g., the existence of a pure condensate or a quasicondensate in a weakly trapped 1D system [2,3], the connection to the concept of a Luttinger liquid [4], and the behavior of density and phase fluctuations [3]. The transfer of a Bose-Einstein condensate into a 1D system as discussed here can thus provide important information, e.g., about the coherence properties associated with the development of phase fluctuations [3]. A wealth of new phenomena is also expected to occur in 1D collisional physics that may be studied in the expansion of a dense ensemble transferred to a 1D or quasi-1D waveguide. Waveguides with high transverse frequencies provide a tool for the experimental realization of famous theoretical models such as a $1 \mathrm{D}$ gas of impenetrable bosons, the so-called Tonks gas $[5,6]$.

In this Rapid Communication we report on the experimental realization of the transfer of BECs into a quasi-1D waveguide created by a blue detuned hollow laser beam. To transfer the atoms coherently we have developed a new type of hybrid trap (Fig. 1). This combined optical dipole and magnetic (DM) trap consists of a waveguide added to the 3D potential of an Ioffe-type magnetic trap. It allows for a natural connection between the magnetic trap and a pure 1D waveguide geometry created by a Laguerre-Gaussian $\left(\mathrm{TEM}_{01}^{*}\right)$ laser beam. In our scheme, we directly obtain BEC by rf evaporation in the DM trap. By subsequently ramping down the magnetic trapping field of the DM trap, the BEC is transferred into the pure optical dipole potential waveguide and its dynamics is observed. In particular the phase coherence of the ensemble at the different stages of this process is investigated with a Bragg interferometer scheme. As a result we find a phase-coherent and mean-field-dominated acceleration in the waveguide on a time scale of $20 \mathrm{~ms}$, much longer than the mean-field release time in the usual 3D expansion of a condensate.
As a class of waveguides, optical dipole potentials formed by blue detuned hollow laser beams are well suited for gaining insight into different radial confinement regimes. By changing the beam parameters they can be tuned from systems with a radial energy-level spacing smaller than the mean-field energy of the ensemble to 1D Tonks gas systems with strong radial confinement. So far these potentials have only been investigated with thermal atomic samples [7].

Optical dipole potentials have previously been used to manipulate BECs. The creation of BECs in a red detuned dipole and magnetic trap [8] and studies of spinor condensates in a 3D red detuned dipole trap [9] as well as the transfer of a BEC into an optical lattice [10] were recently reported. Note, however, that a focused red detuned laser beam creates a 3D trapping potential, whereas the blue detuned hollow $\mathrm{TEM}_{01}^{*}$ laser mode discussed here represents an open 1D waveguide. The radially symmetric intensity distribution, $I(r)$, of a $\mathrm{TEM}_{01}^{*}$ mode is given by

$$
I(r)=\frac{4 P r^{2}}{\pi r_{0}^{4}} e^{-2\left(r^{2} / r_{0}^{2}\right)},
$$

where $P$ and $r_{0}$ are the laser power and the beam waist, respectively. With a power of $P=1 \mathrm{~W}$ at $532 \mathrm{~nm}$ and a beam waist of $r_{0} \approx 10 \mu \mathrm{m}$, a dipole potential at the focal plane with a maximum value of $\sim 120 \mu \mathrm{K} k_{B}$ and a transverse oscillation frequency of $\sim 6 \mathrm{kHz}$ (corresponding to $570 \mathrm{nK}$ ) for ${ }^{87} \mathrm{Rb}$ atoms can be realized. Since the atoms are guided in the low intensity region of the light field, light scattering that leads to decoherence is suppressed for atoms in the transverse ground state by more than three orders of
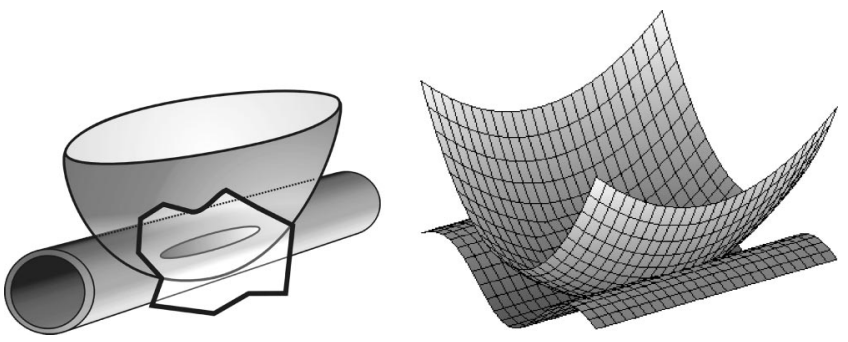

FIG. 1. Schematic view of the potential configuration (left) and magnetic and dipole potential in the matched case (right). 


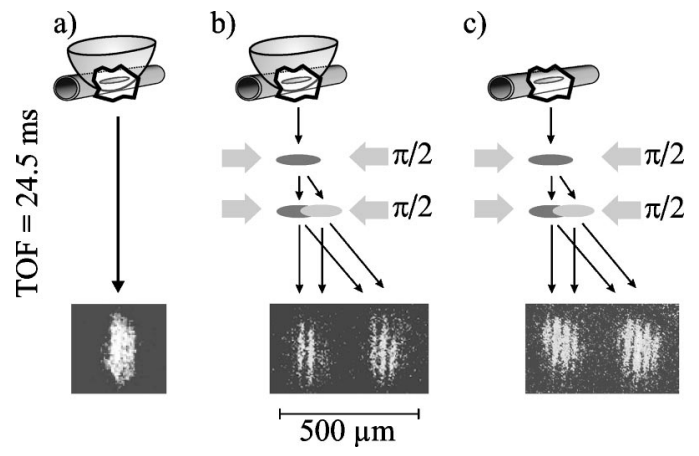

FIG. 2. (a) Absorption image after time of flight for a BEC created in the DM trap. (b) Autocorrelation interferometer scheme and interference fringes for $t_{B}=3 \mathrm{~ms}$ for an ensemble released from the DM trap. (c) Same as (b) for $t_{B}=1 \mathrm{~ms}$ and an ensemble stored in the waveguide for an evolution time of $6 \mathrm{~ms}$.

magnitude in comparison with the scattering rate in the intensity maximum. For our measurements we adjusted the laser parameters such that the mean radial oscillation frequency of $415 \pm 10 \mathrm{~Hz}$ matches the radial confinement of the magnetic trap with an oscillation frequency of 400 $\pm 10 \mathrm{~Hz}$. The waveguide was superimposed on the long axis of the magnetic potential (Fig. 1) resulting in a DM trap with a total radial oscillation frequency of $576 \pm 14 \mathrm{~Hz}$ and an axial oscillation frequency of $14 \mathrm{~Hz}$, solely due to the magnetic potential. The Rayleigh range $R \approx 3.7 \mathrm{~mm}$ of the focused waveguide was much longer than the maximum halfwidth of the observed atomic clouds.

The experimental apparatus was described elsewhere [11] and only the important steps to obtain BEC in the DM trap are given here: Approximately $10^{9}{ }^{87} \mathrm{Rb}$ atoms were collected in a MOT. After their transfer to a cloverleaf magnetic trap [12], the atoms were cooled to a point just above the critical temperature $\left(T \approx 1.5 T_{c}\right)$ by rf induced evaporation. Then the light intensity of the waveguide potential was slowly ramped up within $\approx 15 \mathrm{~ms}$, transferring the ensemble into the DM trap. Subsequently a final rf evaporation ramp was applied, cooling the ensemble below $T_{c}$ and thus creating a Bose-Einstein condensate of up to $2 \times 10^{5}{ }^{87} \mathrm{Rb}$ atoms in the DM trap. Note that the DM trap still allows rf evaporation of atoms via the waveguide axis. We observe a lifetime of the BEC in the DM trap on the order of one second, which is comparable to the BEC lifetime observed in our pure magnetic trap. We observe the usual anisotropic expansion of BECs in a time-of-flight measurement as shown in Fig. 2(a).

To investigate the phase-coherence of the ensemble in the DM trap we use a simple two beam-splitter interferometer scheme. The interferometer consists of only two $\pi / 2$ Bragg pulses separated by a time $t_{B}$, applied after switching off the trapping potentials as indicated in Fig. 2(b). The beam splitters are realized by $\pi / 2$ Bragg pulses $[13,14]$ of counterpropagating laser beams aligned parallel to the axis of the waveguide, which split an incoming wave packet into a coherent superposition of two wave packets with velocities differing by $2 \hbar \mathrm{k} / \mathrm{m}=11.7 \mathrm{~mm} / \mathrm{s}$. The two laser frequencies are detuned by $\approx 6 \mathrm{GHz}$ from the ${ }^{87} \mathrm{Rb}$ line at $780 \mathrm{~nm}$ with a fixed frequency difference set to match the Bragg condition.
The two wave packets created by the first $\pi / 2$-pulse separate in space during the free evolution time $t_{B}$ by an amount $\Delta x=(2 \hbar k / m) t_{B}$. The second $\pi / 2$ pulse then recombines the partially overlapping clouds in both exit ports of the interferometer, which again differ in momentum by $2 \hbar k$. The displacement leads to interference fringes in both exit ports due to the additional phase distribution caused by the expansion of the condensate, similar to the autocorrelation measurements in the $\pi / 2-\pi-\pi / 2$ geometry of [15].

The observed spacing of the interference fringes can be understood as follows: The mean-field repulsion causes a parabolic density distribution of the BEC in a harmonic trap. Once the axial confinement is removed, the condensate expands mainly due to the conversion of mean-field energy into kinetic energy. Since the mean-field energy $U_{m f}$ is proportional to the parabolic density distribution, the local acceleration $\dot{v} \propto \nabla U_{m f}$ depends linearly on position. As a consequence the ensemble keeps a parabolic density distribution during expansion while acquiring a velocity field that linearly increases with position, $v(x)=\alpha(t) x$. The velocity gradient is given by $\alpha(t)=\dot{\lambda}(t) / \lambda(t)$ with the scaling parameter $\lambda(t)$ defined in [16] indicating the size of the condensate relative to its size in the trap. The local velocity difference between the two overlapping clouds thus does not depend on position but only on the displacement and on time $\Delta v$ $=\alpha(t) \Delta x$. The observed interference pattern therefore corresponds to the interference of two plane matter waves with a relative velocity $\Delta v$. The corresponding fringe spacing $d$ $=h / m \Delta v$ can be used to deduce the velocity gradient of the ensemble

$$
\alpha(t)=\frac{h}{m d \Delta x} .
$$

Note that the above discussion assumes that all mean-field energy is converted to kinetic energy before the interferometer sequence. In our experiment the time of flight prior to the first interferometer pulse is chosen to be $2 \mathrm{~ms}$ in which more than $95 \%$ of the mean-field energy is converted into kinetic energy.

We now turn to our measurements of phase coherence for 3D ensembles in the DM trap and during expansion in the quasi-1D waveguide. The quasi-1D regime is characterized by a chemical potential only a few times larger than the ground-state energy of the potential [17]. We obtained the interference pattern of the BEC produced in the DM trap by taking absorption images of the density distribution along an axis orthogonal to the Bragg laser pulses after the interferometer sequence and a total time of flight of $24.5 \mathrm{~ms}$ [Fig. $2(\mathrm{~b})]$. The time between the pulses was varied from $t_{B}=1$ to $4 \mathrm{~ms}$, resulting in a displacement of the interfering clouds of $\Delta x=12$ to $48 \mu \mathrm{m}$. The largest displacements roughly correspond to half the condensate size of $\approx 100 \mu \mathrm{m}$. For all these displacements high-contrast, equally spaced interference fringes were observed, demonstrating both the coherence of the ensemble on these length scales and the validity of the above model for the 3D BECs in the DM trap. 


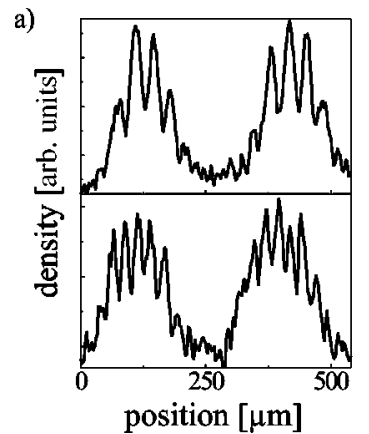

b)

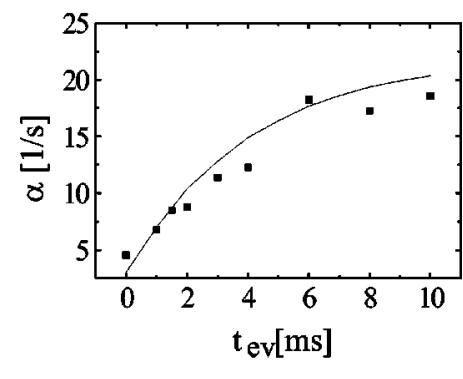

FIG. 3. (a) Interference signals from guided ensembles for evolution times of $4 \mathrm{~ms}$ (top) and $6 \mathrm{~ms}$ (bottom) in the waveguide. (b) Velocity gradient versus guiding time deduced from measurements of the fringe spacing (dots) in comparison to the numerical calculation (solid line).

In a further set of experiments, the transfer of the BoseEinstein condensed ensemble to the waveguide and the subsequent evolution of the atomic ensemble inside the waveguide were investigated. Again, in a series of measurements the $\pi / 2-\pi / 2$ pulse interferometer scheme was now applied to an ensemble transferred from the DM trap into the waveguide for different evolution times $t_{e v}$ in the waveguide. For this purpose the natural connection of the DM trap to the waveguide was demonstrated by lowering the magnetic field to zero over a time of typically $25 \mathrm{~ms}$, thus releasing the ensemble into the waveguide. Figure 2(c) shows an example of the resulting interference fringes. Figure 3(a) shows examples of cross sections through the interference signals. For these measurements the time between the Bragg pulses was set to $1 \mathrm{~ms}$, resulting in a displacement of the wave packets of $\approx 12 \mu \mathrm{m}$. To our knowledge, these interference signals document for the first time the axial phase coherence of an ensemble after the transfer of a BEC into a quasi-1D waveguide and a free 1D evolution.

As another important result, the equal spacing of the interference fringes confirms the linear velocity distribution predicted by the model given above [Eq. (2)] for the 1D expansion in the waveguide.

The decrease of fringe spacing for increased evolution times in the waveguide demonstrates an increasing velocity spread of the wave packet as mean-field energy is converted into kinetic energy within the waveguide. We observe a decrease in fringe spacing with time even for evolution times of $10 \mathrm{~ms}$ in the waveguide, i.e., the atoms are still accelerated by the mean-field energy. These measurements clearly show that the conversion of mean-field energy into kinetic energy is more than one order of magnitude slower than in $3 \mathrm{D}$ due to the reduced dimensionality inside the waveguide.

From the regular fringe spacing we deduced the spatial velocity gradient of the ensemble for different evolution times. Figure 3(b) compares these data to the result of a numerical calculation of the parameter $\alpha(t)$ according to [16]. Note that the calculation does not include any free parameters, or depend on the particle number. The good agreement clearly demonstrates the consistency of the ensemble dynamics with a mean-field dominated and phase-coherent expansion inside the waveguide and the applicability of the scaling laws to the quasi-1D regime.

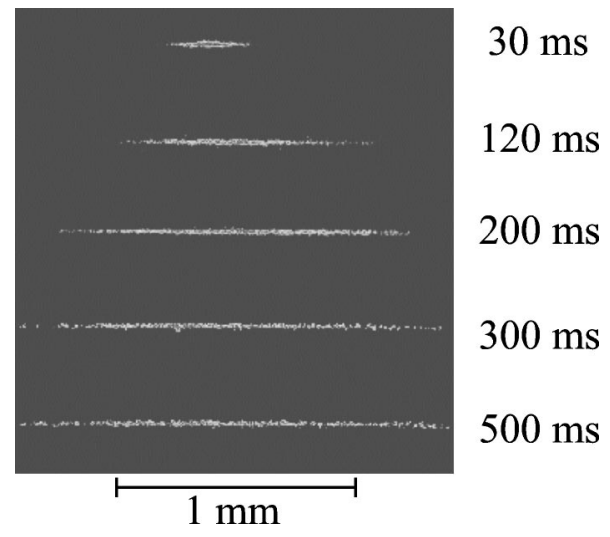

FIG. 4. Atoms from a BEC loaded to a doughnut waveguide with different evolution times inside the waveguide.

The above interferometer scheme is well suited to study the axial expansion of the ensemble inside the waveguide within the first $10 \mathrm{~ms}$. For longer guiding times, when the ensemble density is significantly reduced we have directly studied the expansion by absorption imaging without an additional time of flight. Figure 4 shows examples for these measurements for evolution times up to $500 \mathrm{~ms}$. On time scales above $40 \mathrm{~ms}$ the conversion of mean-field energy into kinetic energy is nearly complete and the ensemble is expected to expand with constant velocity keeping its parabolic density distribution. We indeed found the predicted parabolic density distribution confirmed for evolution times up to $150 \mathrm{~ms}$ from cross sections through images as shown in Fig. 4. These measurements are limited only by our field of view, as no reliable fits to the data can be obtained as soon as the imaged length of the ensemble corresponds to the size of our charge-coupled-device array.

An estimate of the maximum velocity at the edge of the parabolic density distribution after an expansion in the waveguide can be obtained as follows: After long expansion times in the waveguide the ensemble width is much larger than its initial width. In this limit the maximum velocity at the edge of the cloud can be calculated from simple kinematics and is given by $v_{\max }=\sqrt{10 E_{k i n} / m}$ where $E_{k i n}$ is the kinetic energy per particle. If all internal energy is converted in kinetic energy, $E_{k i n}$ can be replaced by the initial internal energy $E_{i n t}$.

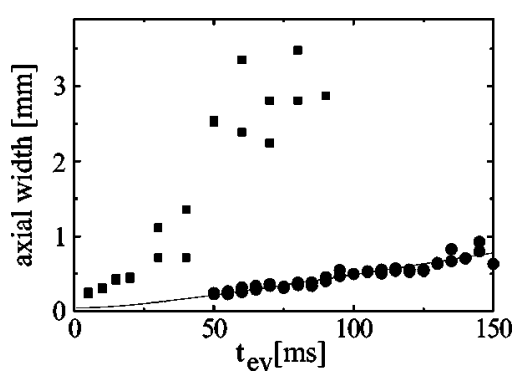

FIG. 5. Expansion of BECs loaded into the waveguide. Squares: Gaussian half-width of ensembles rapidly transferred from the magnetic trap. Circles: Thomas-Fermi half-width of ensembles adiabatically transferred from the DM trap. Solid line: theoretical prediction for $N=5 \times 10^{4}$ atoms. 
The internal energy per particle in the Thomas-Fermi limit is well known [18] and is given by $E_{\text {int }}=0.46 k_{B} N^{2 / 5} \mathrm{nK}$ for our experimental parameters. For $N=5 \times 10^{4}$ atoms we find $v_{\max }=5.8 \mathrm{~mm} / \mathrm{s}$, in good agreement with the velocity deduced from our experimental data $v_{\max }=5.9 \mathrm{~mm} / \mathrm{s}$. This again confirms the mean-field-dominated expansion of the cloud in the waveguide.

Finally, we have compared different loading mechanisms of the waveguide. Figure 5 shows a comparison of the expansion of atomic clouds loaded from the DM trap to the expansion of clouds loaded directly from a BEC created in the cloverleaf magnetic trap. The latter was done by instantly switching on the doughnut beam and switching off the magnetic trap in $200 \mu \mathrm{s}$. For direct loading from the magnetic trap, the system expands to several millimeters in length within $\approx 100 \mathrm{~ms}$, which indicates the nonadiabaticity of the direct transfer process. In contrast, the cloud's expansion for loading from a BEC first created in the DM trap shows excellent agreement with the theoretical prediction [16] of mean-field-dominated expansion (solid line in Fig. 5). In this case the atomic density distribution spreads out over an axial region of $\approx 1 \mathrm{~mm}$ within $150 \mathrm{~ms}$, and the cigar-shaped BEC transforms to a straight hair of quantum gas.

In conclusion we have presented three important results, paving the way to study $1 \mathrm{D}$ quantum gases. First, we have observed Bose-Einstein condensation of an ensemble of ${ }^{87} \mathrm{Rb}$ atoms in a hybrid trap realized by the combined potential of an Ioffe-type magnetic trap and a blue detuned dipole waveguide potential. Second, the coherence of the ensemble in the trap and in the waveguide was measured with a simple autocorrelation interferometer scheme. Third, we investigated the transfer process of Bose-Einstein condensates into a blue detuned dipole waveguide and studied the subsequent evolution of the ensemble in a quasi-1D waveguide. In these experiments we demonstrated that a fully coherent transfer is possible by our scheme and observed a mean-fielddominated expansion of the ensemble for adiabatic loading conditions.

These investigations open a path for future studies of the different regimes of $1 \mathrm{D}$ quantum gases. The realization of a Tonks gas of impenetrable Bosons seems feasible in our geometry. After loading, the dipole waveguide potential can be increased adiabatically to reach the 1D regime in which transverse degrees of freedom are fully frozen out $\left(\omega_{r}\right.$ $\approx 10 \mathrm{kHz}$ ). As additional magnetic fields can be superimposed on the dipole potential, the scattering length $a$ can be tuned using Feshbach resonances to achieve a suitable value for the Tonks gas regime. Also, e.g., spinor condensates [9] and dark solitons $[19,20]$ can be investigated in 1D waveguide geometries. The waveguide can easily be closed by additional light field mirrors [11] or a superimposed weak longitudinal trapping potential. This opens opportunities for a first realization of a guided matter wave interferometer.

This work was supported by SFB 407 of the Deutsche Forschungsgemeinschaft.
[1] M. J. Anderson et al., Science 269, 198 (1995); K. B. Davis et al., Phys. Rev. Lett. 75, 3969 (1995); C. C. Bradley et al., ibid. 78, 985 (1997); D. Fried et al., ibid. 81, 3811 (1998).

[2] N. van Druten and W. Ketterle, Phys. Rev. Lett. 79, 549 (1997).

[3] D. Petrov, G. V. Shlyapnikov, and J. Walraven, Phys. Rev. Lett. 85, 3745 (2000), and references therein.

[4] H. Monien, M. Linn, and N. Elstner, Phys. Rev. A 58, R3395 (1998).

[5] L. Tonks, Phys. Rev. 50, 955 (1936).

[6] M. Olshanii, Phys. Rev. Lett. 81, 938 (1998).

[7] T. Kuga et al., Phys. Rev. Lett. 78, 4713 (1997), and references therein.
[8] D. Stamper-Kurn et al., Phys. Rev. Lett. 80, 2027 (1998).

[9] J. Stenger et al., Nature (London) 396, 345 (1998).

[10] B. Anderson and M. Kasevich, Science 282, 1686 (1998).

[11] K. Bongs et al., Phys. Rev. Lett. 83, 3577 (1999).

[12] M.-O. Mewes et al., Phys. Rev. Lett. 77, 416 (1996).

[13] J. Stenger et al., Phys. Rev. Lett. 82, 4569 (1999).

[14] E. W. Hagley et al., Phys. Rev. Lett. 83, 3112 (1999).

[15] J. Simsarian et al., Phys. Rev. Lett. 85, 2040 (2000).

[16] Y. Castin and R. Dum, Phys. Rev. Lett. 77, 5315 (1996).

[17] A. E. Muryshev et al., Phys. Rev. A 60, R2665 (1999).

[18] G. Baym and C. Pethick, Phys. Rev. Lett. 76, 6 (1996).

[19] J. Denschlag et al., Science 287, 97 (2000).

[20] S. Burger et al., Phys. Rev. Lett. 83, 5198 (1999). 